\title{
CURRÍCULO, PRÁXIS E HORIZONTES DE FORMAÇÃO: política de formação de professores na província de Buenos Aires (2004-2007)
}

Myriam Southwell

CONICET/Universidad Nacional de La Plata

Noralí Boulan

Universidad Nacional de La Plata

\begin{abstract}
Resumo
Este artigo pretende analisar a política de formação de professores realizada na província de Buenos Aires entre 2004 e 2007, através da análise dos primeiros desenvolvimentos curriculares, dos debates realizados em diferentes espaços institucionais e, finalmente, da aprovação do novo desenho curricular para formação de professores. Estamos interessados em descrever como o novo desenho curricular buscou o desenvolvimento do professor como trabalhador e produtor cultural, sujeito político, com perspectivas crítico-transformadoras e alto compromisso com suas comunidades e com a educação pública. Para isso, procurou desenvolver horizontes de formação levando em consideração o professor não apenas como profissional de ensino, mas também como pedagogo e como trabalhador da cultura.
\end{abstract}

Palavras-chave: Currículo, horizontes político-culturais, práxis

\begin{abstract}
This article aims to analyze the teacher training policy of carried out in province of Buenos Aires between 2004 and 2007, through the analysis of the first curricular documents, debates in different institutions and - finally - approval of the new curriculum for teacher training. We are interested in describing how the new curricular design sought teaching work as a cultural producer, political subject, withcritical-transformative perspectives and highcommitment to their communities and public education. To do this, he sought to deploy training horizons taking into consideration the teacher not only as a teaching profesional but also as a pedagogue and as a culture worker.
\end{abstract}

Keywords: Curriculum, political-cultural horizons, práxis

\section{Resumen}

Este artículo se propone analizar la política de formación de docentes llevada adelante en la provincia de Buenos Aires entre los años 2004 y 2007, a través del análisis de los primeros desarrollos curriculares, los debates realizados en distintos espacios institucionales y -finalmenteaprobación del nuevo diseño curricular para la formación de docentes. Nos interesa describir como el nuevo diseño curricular buscó el desarrollo docente como trabajador cultural, como productor cultural, sujeto político, con perspectivas crítico-transformativas y alto compromiso con sus comunidades y la educación pública. Para ello, buscó desplegar horizontes formativos tomando en consideración al maestro/a no sólo como profesional de la enseñanza, sino también como pedagogo y como trabajador de la cultura.

Palabras clave: Curriculum, horizontes político-culturales, praxis 
En Argentina, la afirmación de la educación como derecho social y una mayor principalidad estatal fue impulsada en términos legislativos por la sanción de la Ley de Educación Nacional (en adelante, LEN) en 2006. La mayoría de las normas sancionadas entre 2005 y 2015, en un contexto de revisión y crítica de los gobiernos neoliberales de décadas anteriores, contienen afirmaciones que dan cuenta de la principalidad del Estado respecto de la responsabilidad por la educación, vinculándola a la concepción de la educación como derecho social. Esta principalidad estatal contrasta con las orientaciones hacia la subsidiariedad del Estado que caracterizaron las políticas de la dictadura hacia la educación, que no se modificaron significativamente durante la década de 1980 y acentuadas por las leyes sancionadas en los '90 (incluso desde la dictadura). Este artículo busca ocuparse específicamente de la política de formación de docentes llevada adelante en la provincia de Buenos Aires entre los años 2004 y 2007, a través del análisis de los primeros desarrollos curriculares, los debates realizados en distintos espacios institucionales y -finalmenteaprobación del nuevo diseño curricular para la formación de docentes.

\section{Las políticas educacionales en el período 2003-2015: reposicionando el rol regulatorio del Estado}

Los modos en que se plantearon los vínculos entre escolarización e igualdad fueron revisados y reformulados por la política educativa impulsada a partir de 2003, cuando en Argentina se inició el gobierno de Néstor Kirchner. Tal reformulación se dio en torno de la equivalencia discursiva igualdad-inclusión educativa, que dio lugar a dos cadenas de significantes emparentadas. Una de ellas asoció la igualdad y la inclusión educativas a la afirmación de la educación como derecho social, la principalidad de la acción estatal, la reconstrucción de lo común y la consideración de la diversidad; mientras que la otra enlazó la igualdad y la inclusión educativas a la idea de centralidad de la enseñanza, el trabajo con situaciones de desigualdad educativa -tales como la sobredad y la repitencia- y de vulnerabilidad social, y la promoción de modos organizacionales alternativos para las instituciones escolares.

La LEN trajo consigo una serie de revisiones y modificaciones de principios: estableció que la educación y el conocimiento son un bien público y un derecho personal y social garantizados por el Estado. Éste asume un papel principal en asegurar el acceso de todos los ciudadanos y ciudadanas a la información y al conocimiento como instrumentos centrales de la participación en un proceso de desarrollo con crecimiento económico y justicia social. Luego de su dilución en el último tercio del siglo XX, la noción de igualdad aparece en el nuevo cuerpo normativo con diversas expresiones que también es posible observar en el contexto latinoamericano. De manera específica, en el caso argentino la igualdad se inscribe como igualdad de oportunidades y se asocia a los intentos por instituir algo del orden de lo común y que tiene expresiones en retóricas universalistas.

En este marco, las políticas que la LEN denomina "de promoción de la igualdad educativa" aparecen asociadas al enfrentamiento de situaciones de injusticia, marginación, 
estigmatización y otras formas de discriminación, derivada de factores socioeconómicos, culturales, geográficos, étnicos, de género o de cualquier otra índoles, que afecten el ejercicio pleno del derecho a la educación (LEN art. 79). Estas políticas también se presentan ligadas a la inclusión, reconocimiento e integración de todos/as los/as niños/as, jóvenes y adultos en todos los niveles y modalidades, principalmente los obligatorios, enfatizando la necesidad de garantizar la "igualdad de oportunidades y resultados educativos" y la provisión de recursos materiales y pedagógicos a aquellos alumnos/as, familias y escuelas que se encuentren en situación socioeconómica desfavorable (LEN art. 80). En este sentido, se establece la promoción de la inclusión de niños/as no escolarizados/as en espacios escolares no formales como tránsito hacia procesos de reinserción escolar plenos (LEN art. 83).

Siguiendo y profundizando estos parámetros, en 2009 el Consejo Federal de Educación aprobó el Plan Nacional de Educación Obligatoria, en el que se plasman una serie de significados en torno de la idea de inclusión que venía desplegando el discurso oficial nacional en los últimos años. Se trató de un plan trienal que abarcaba la educación obligatoria definida por la Ley de Educación Nacional: la sala de cinco años, la educación primaria y la educación secundaria con todas sus modalidades. Allí se plantean una serie de problemas y desafíos relativos a los diferentes niveles y vinculados con la cuestión de la igualdad, presentada como una línea de política educativa. En relación al nivel primario, se señala la necesidad de completar el acceso al nivel; de ampliar la cobertura de las zonas rurales y avanzar con modelos organizativos alternativos y agrupamientos más flexibles; de mejorar las tasas de escolarización; de ampliar el acceso de adolescentes, jóvenes y adultos que "abandonaron" los estudios primarios o están en situación de encierro, a través de programas que permitan el cumplimiento de la obligatoriedad; de mejorar, con "urgencia" el acceso y permanencia en el nivel de niños, niñas y jóvenes pertenecientes a diferentes etnias aborígenes, contando con maestros interculturales y bilingües; de que los docentes con mayor experiencia y calificación se desempeñen en las escuelas más vulnerables, siguiendo lo establecido por la Ley de Educación Nacional (Ministerio de Educación de la Nación, 2009). Asimismo, el Plan vinculó la noción de igualdad a la posibilidad de reversión de algunos indicadores relativos a situaciones de "abandono" y repitencia escolar. Junto con ello, se puso un fuerte énfasis en la "permanencia con calidad y la finalización del nivel, sobre todo en sectores más desfavorecidos".

En ese discurso, el contexto de desigualdad social que suponían grupos excluidos de la escolarización requirieron la consolidación de políticas que avanzaran en la "inclusión y retención" de niños, niñas, adolescentes y jóvenes que aún permanecían fuera del sistema educativo. En este marco, la formación docente inicial y continua aparecía como un factor clave de la calidad de la educación. También se liga a la extensión de la jornada y a la universalización de la educación escolar para niños/as de cuatro años de edad, priorizando a los sectores más desfavorecidos (LEN art. 135) como modo de favorecer la inclusión educativa. Asimismo, el cuerpo normativo vinculó explícitamente el trabajo docente al logro de mayores condiciones de igualdad, por lo que la LEN instituyó la capacitación y actualización docente integral, gratuita y en servicio a lo largo de toda la carrera (LEN art. 67). 
Como puede observarse, la inclusión fue significante central que organizó los significados puestos a circular por las definiciones de política educativa (Southwell y Vassiliades, 2016). La noción de inclusión comienza a conformarse durante este periodo, en el significante vacío donde es posible observar que la cadena equivalencial que lo configura se despliega a través de un conjunto de "significantes flotantes" (Laclau, 1996) que respondieron a demandas sociales, políticas y culturales que supusieron la configuración hegemónica de un discurso que apelaba a la ampliación de derechos de amplios sectores sociales. Una condición de posibilidad para que esto sucediera fue la aparición de un elemento nuevo, es decir, una diferencia que no logra articularse discursivamente en el marco de las políticas educativas de periodos históricos anteriores. El cumplimiento de la obligatoriedad escolar, la incorporación de modalidades de educación intercultural y en contextos de encierro, la integración de sujetos que transitan por los llamados "espacios no formales", la cobertura en zonas rurales, que los maestros con mayor experiencia trabajen en las zonas más vulnerables, son todas aspiraciones fundadas en la premisa de la inclusión escolar, asociada a la idea de igualdad y sustentada en una retórica universalista. En este marco debe leerse también la implementación de la Asignación Universal por Hijo. Originada en octubre de 2009, constituyó una política del Estado Nacional que otorga una prestación no contributiva similar a la que reciben los hijos de los trabajadores formales a aquellos niños, niñas y adolescentes residentes en la República Argentina, que no tengan otra asignación y pertenezcan a grupos familiares que se encuentren desocupados o se desempeñen en la economía informal. Se otorgó a todo ciudadano/a que cumpla con los requisitos básicos, estando su pago condicionado a la acreditación del cumplimiento de la escolaridad obligatoria, controles de salud y vacunación. De modo general, estas medidas, han representado la reversión de algunas de las políticas de los 90 en un contexto de significativo crecimiento económico. Ambas administraciones han adoptado un discurso que enfatiza la intervención del Estado y los derechos sociales y que en el plano pedagógico se despliega a partir del par igualdad-inclusión (Southwell y Vassiliades, 2016).

\section{Acerca de la "centralidad de la enseñanza": notas sobre un modo de pensar la inclusión}

Desde el punto de vista de la discursividad oficial, la implementación del Programa Integral para la Igualdad Educativa (PIIE) por parte del Ministerio de Educación de la Nación a partir del año 2004 constituyó una de las vías principales a través de la cual se redefinieron los términos en que se había planteado la relación entre educación e igualdad en las reformas de la década del '90 en nuestro país. Dirigido al nivel primario y específicamente a sectores en condiciones de vulnerabilidad social, el PIIE presentó algunas notas distintivas respecto de las políticas focalizadas desplegadas en la mencionada década y puso a circular sentidos y significados diferentes para la escolarización y el trabajo docente. Entre ellos, cabe destacar el papel principal asignado al Estado en garantizar el derecho a la educación de las nuevas generaciones, y la apuesta a la igualdad de oportunidades educativas como una dimensión 
constitutiva de la igualdad social, asociado a fortalecer los procesos de enseñanza y aprendizaje, reposicionándolos y ubicándolos en el centro de la tarea escolar cotidiana.

Comprendió actividades de apoyo a las iniciativas emanadas de las comunidades educativas, para lo que ofrecía acompañamiento pedagógico y financiero a través de un subsidio, como así también recursos e instancias de formación docente, el impulso al establecimiento de vínculos entre la escuela y la comunidad, el reparto de recursos materiales para las escuelas, como asimismo para el mejoramiento de la infraestructura escolar. En torno de la idea de "reposicionar la enseñanza", la propuesta del PIIE pone en relación la realización del derecho a la educación con la distribución de bienes y el mejoramiento de las condiciones materiales como una de las vías para alcanzar la igualdad de oportunidades, asociada a la igualdad social.

Así, la noción de centralidad de la enseñanza estructuró el discurso pedagógico oficial nacional desde 2004 a 2015, estando estrechamente emparentada a la idea de inclusión y la reposición del valor de la igualdad. Ellos se asociaron a una serie de ideas relativas a la construcción de agrupamientos institucionales más flexibles y al fortalecimiento de la autoridad adulta y el cuidado de las nuevas generaciones, como modos de producir respuestas a situaciones de sobreedad, repitencia y exclusión escolar consideradas de desigualdad educativa. La noción de igualdad, por su parte, se tornó equivalente de la de inclusión y se emparentó a la principalidad estatal, la afirmación del derecho social de la educación y la restitución de lo común, cuestión que se expresó, fundamentalmente, en el tratamiento del tema de la diversidad, la promoción de otras modalidades educativas, la implementación de la Asignación Universal por Hijo y el establecimiento del carácter "nacional" de la educación y del piso común de aprendizajes para todos/as los/as estudiantes.

\section{Las políticas educativas en la provincia de Bunos Aires: la gran consulta bonaerense}

En consonancia con los planteamientos desarrollados a nivel nacional, en el ámbito bonaerense la Ley Provincial de Educación, [en adelante, LPE] sancionada en 2007 establece y reconoce como responsabilidad indelegable del Estado el derecho a la educación. De este modo, en su artículo 6 plantea la responsabilidad indelegable del Estado en materia educativa. Las máximas autoridades educativas de la provincia fueron durante este período: Mario Oporto (2001-2005); Adriana Puiggrós (2005-2007) y nuevamente Mario Oporto (20072011).

En ese marco se desarrolló durante los años 2004 y 2005 en el ámbito de la Provincia de Buenos Aires, un proceso de consulta social en relación a la educación, la formación docente y el rol social de la escuela. Así lo describe quien fuera Subdirectora y Directora de la Dirección de Educación Superior de la Provincia de Buenos Aires (2009-2015) durante la reformulación e implementación curricular para el Profesorado de Nivel Primario: 
"En la Provincia de Buenos Aires en el Nivel de Educación Superior se inició un proceso de democratización de las instituciones y de la construcción del conocimiento y del saber. Provincia de Buenos Aires empieza a tomar contacto con autoridades a nivel nacional inclusive con diferentes instancias a nivel gubernamental y sindical para analizar cuál era el estado de situación de la formación docente, qué nivel de impacto tenía esa segmentación y fragmentación de la formación, fundamentalmente en los Profesorados de Educación Inicial y Primaria. Ese fue uno de los ítems que se tuvo en cuenta como indicador de este proceso de construcción y de reformulación del Profesorado de Educación Primaria.

Se inicia entonces un proceso de consulta provincial sobre cuáles serían los horizontes acerca de qué escuelas y qué docentes eran necesarios. Se buscaba tener en cuenta desde el gobierno de la gestión central [la opinión de] la sociedad bonaerense en su conjunto. Se produjo una consulta de carácter abierta a diferentes referentes, no solamente del sistema educativo sino desde la sociedad en su conjunto, como fueron diferentes organizaciones sociales, barriales, bibliotecas populares, como proceso de consulta. Después con determinados actores del sistema educativo provincial.

Por lo tanto, se inicia el proceso de consulta -con instrumentos como talleres, entrevistas- a otras organizaciones y al propio sistema. Se tuvo en cuenta por supuesto a directores y maestros de escuelas de la provincia con la característica de la complejidad y diversidad que tiene. Y desde norte a sur, de este a oeste de la Provincia de Buenos Aires, con el universo de escuelas primarias que la provincia tiene, se trabajó en talleres, en entrevistas personales con directores y maestros, también con supervisores que en ese momento todavía eran areales; antes de la ley no existían los inspectores jefes distritales y regionales. (...) Ese fue el primer proceso" (Entrevista a Directora de Educación Superior).

Es posible destacar a partir de este testimonio que uno de los sentidos vinculados a la inclusión tuvo vinculación con participación, con la "democratización y/o apertura" de los sentidos del sistema educativo y el reconocimiento de la diversidad en todos los aspectos del término: social, cultural, económica.

De modo similar, en la búsqueda de un profundo involucramiento de los sujetos destinatarios de dicha política, se realizaron también procesos de consulta a los Institutos Superiores de Formación Docente, [ISFD], se desarrollaron reuniones, convocatorias, jornadas de reflexión institucionales, en los cuales se apuntaba a generar y promover la participación de los sujetos en la reformulación curricular. Las entrevistas realizadas a funcionarias de gobierno, a asesoras curriculares convocadas para dicho fin, a docentes y directivos de diversos ISFD de la Provincia de Buenos Aires, así como la recuperación de algunas ideas centrales de Jorge Huergo en tanto Director de Educación Superior de la Dirección General de Cultura y Educación de la Provincia de Buenos Aires, permiten dar cuenta de los mismos, sus disputas y sus tensiones.

En este sentido, el Marco General de la Política Curricular (MGPC) explicita la serie de acciones promovidas por el Estado provincial para desarrollar y orientar dicha consulta: 
"Los diseños surgen de la producción de equipos técnicos en consulta con los/as docentes (profesores/as, equipos directivos e inspectores/as) de los respectivos niveles. El proceso de revisión y elaboración contó con numerosos aportes provenientes de diferentes actores del sistema educativo y de la comunidad, quienes no sólo facilitaron insumos para el análisis, sino también para la validación de los primeros documentos elaborados. Entre estos aportes, que constituyen los antecedentes de los diseños curriculares, se destacan:

- Ronda de Consultas 2004. El futuro de la educación: tanto docentes como alumnos/as y sus familias participaron a través de la respuesta a un cuestionario.

- Plan Provincial de Consulta 2006 sobre la nueva Ley de Educación Nacional: en el marco de la iniciativa del Ministerio de Educación, Ciencia y Tecnología de la Nación, la provincia de Buenos Aires impulsó y sistematizó una amplia consulta, utilizando múltiples estrategias dirigidas a diferentes actores, entre las que pueden destacarse: publicación de artículos para el debate, recepción y análisis de documentos elaborados por diversas organizaciones públicas y privadas, reuniones de trabajo con representantes de distintos sectores involucrados, jornadas de discusión regionales, jornadas institucionales, encuestas a alumnos/as, familias y foros juveniles en el portal ministerial.

- Plan de Consulta 2007. Nueva Ley Provincial de Educación: se desarrolló una amplia campaña de difusión mediática, se distribuyeron documentos destinados a las instituciones educativas, se organizaron jornadas institucionales y reuniones con todos los miembros del sistema educativo y con grupos de interés (gremios; organizaciones políticas, culturales, sociales y comunitarias; asociaciones estudiantiles; organismos de Derechos Humanos; cámaras empresariales; organizaciones de la producción y el trabajo; credos religiosos; rectores/as de universidades nacionales y privadas con asiento en la Provincia; colegios profesionales).

- Materiales de desarrollo curricular que introdujeron en los distintos niveles y modalidades enfoques conceptuales y orientaciones didácticas que implicaron revisiones de los diseños en vigencia.

- Informes de resultados de los operativos de evaluación y de investigaciones cualitativas elaboradas por los equipos técnicos de la DGCyE.

- Experiencias innovadoras realizadas fundamentalmente en el marco de distintos programas educativos que se implementaron durante los últimos años en las instituciones del sistema.

- Informes de supervisión que dieron cuenta del tratamiento institucional de materiales de desarrollo curricular, así como de las experiencias innovadoras.

- Observaciones y relevamientos de los Equipos Técnicos Regionales en el marco de los encuentros de capacitación docente.

- Encuentros provinciales, regionales y distritales con representantes del sistema educativo organizados por las Direcciones Provinciales de nivel.

- Visitas a instituciones educativas de autoridades y equipos técnicos de la DGCyE. Estas valiosas contribuciones confirmaron la necesidad de producir nuevos diseños curriculares. (DGCyE, MGPC: 10). 


\title{
El diseño curricular de la formación docente
}

A partir de lo enunciado por los Lineamienos Curriculares Nacionales (LCN), el gobierno de la provincia de Buenos Aires desarrolló el proceso de redefinición curricular que permitirán la formulación de los Diseños Curriculares (DC) para los Profesorados de Educación Primaria, Educación Inicial y Educación Física. En esa política curricular se retoman los lineamientos de la Ley de Educación Provincial en relación a prescribir la enseñanza de contenidos educativos, social y científicamente pertinentes. Para ello, la Dirección General de Cultura y Educación elaboró “diseños, propuestas y materiales de desarrollo curricular” (DGCyE, MGPC, 2007:10). Así, el Marco General de la Política Curricular (2007) establece el Estado en relación a la construcción de lo social:

\begin{abstract}
"Algunas representaciones describen al Estado como una totalidad homogénea, como una entidad aislada, separada y distante de la sociedad, que lleva adelante un proyecto uniforme. Sin embargo, esta concepción resulta insuficiente para explicar la complejidad del Estado. En esta perspectiva, éste constituye una construcción social y, por lo tanto, contiene la conflictividad y las diferencias presentes en la comunidad. Es conveniente pensar las políticas estatales como posicionamientos que involucran distintos proyectos e intereses que se dan en el marco de procesos sociales más amplios, en lugar de entenderlas como discursos y acciones unívocas.

El Estado, además, como modo de organización jurídica de los ciudadanos/as para la consecución de sus proyectos de vida, es quien debe garantizar los derechos de todos los sujetos. [...] Ante la preponderancia de las acciones focalizadas, impulsadas en la década de 1990, los diseños y propuestas curriculares constituyen un componente significativo de las políticas públicas universales en materia educativa, concebidas como herramientas para hacer efectiva la igualdad y la inclusión." (DGCyE, DES, MGPC, 2007: 12).
\end{abstract}

Los DC de Formación Docente establecieron la extensión de la carrera de Profesorado en Educación Primaria a cuatro años, lo cual posiciona a sus graduados en igualdad de condiciones en relación a los graduados universitarios ante la posibilidad de acceder a posgrados universitarios; la creación de un espacio curricular, los "Talleres de Integración Interdisciplinarios" (TAIN) que se configuraron como un espacio donde cada institución formadora dispone en forma mensual de cuatro horas con el propósito de discutir diversas problemáticas político-pedagógicos con la participación de profesores y de docentes en formación de cada institución y cuyo eje estructural es el "Campo de la Práctica Docente" y las "Herramientas de la Práctica" correspondientes a cada año de la carrera. Estos espacios de intercambio surgieron por la necesidad de unificar la histórica atomización y fragmentación de las diversas cátedras, de modo de pensar la formación como una totalidad, con visión crítica y con la intención de favorecer la participación democrática, donde, 
particularmente los estudiantes pudieran expresar, debatir y consensuar la propia formación que estaban recibiendo.

Así se encuentra expresado en el anexo de la Resolución 24 del Consejo Federal de Educación del 2007, que establece los LCN, detallan los fundamentos político-pedagógicos que configuran dicha política educativa, formulan referencias al rol docente la importancia de la actualización y desarrollo de una formación que apunte a la criticidad y autonomía de los maestros, donde se comprende el ejercicio de la docencia desde una perspectiva política, reflexiva, crítica y propositiva:

25.1. La docencia como práctica de mediación cultural reflexiva y crítica, caracterizada por la capacidad para contextualizar las intervenciones de enseñanza en pos de encontrar diferentes y mejores formas de posibilitar los aprendizajes de los alumnos y apoyar procesos democráticos al interior de las instituciones educativas y de las aulas, a partir de ideales de justicia y de logro de mejores y más dignas condiciones de vida para todos los alumnos

25.2. La docencia como trabajo profesional institucionalizado, que se lleva a cabo en las instituciones educativas, en el marco de la construcción colectiva de intereses públicos, de aspiraciones compartidas y del derecho social a la educación. Ello implica la necesaria autonomía y responsabilidad profesional para la genuina toma personal de decisiones para enseñar, como una actividad comprometida, enriquecedora y para construir espacios de trabajo compartido y colaborativo en las instituciones escolares en las que el trabajo del equipo docente pueda primar por sobre el trabajo individual y aislado. Requiere, asimismo, del ejercicio de la autoridad pedagógica, no como autoridad formal, sino como profesional reconocido y legitimado por su responsabilidad en la enseñanza y sus propuestas educativas.

25.3. La docencia como práctica pedagógica construida a partir de la transmisión de conocimientos y de las formas apropiadas para ponerlos a disposición de sus alumnos y que toma a la diversidad como contexto. Ello implica la capacidad de analizar la práctica cotidiana incorporando las dimensiones siempre particulares del contexto de la práctica tanto a nivel organizacional como del aula, en vistas a la mejora continua de la enseñanza. Estas prácticas requieren interrogarse acerca de la contextualización de los principios generales de la enseñanza en los espacios locales de su realización.

25.4. La docencia como práctica centrada en la enseñanza implica capacidad para:

- dominar los conocimientos a enseñar y actualizar su propio marco de referencia teórico

- adecuar, producir y evaluar contenidos curriculares

- reconocer el sentido educativo de los contenidos a enseñar

- ampliar su propio horizonte cultural más allá de los contenidos culturales imprescindibles para enseñar en la clase

- identificar las características y necesidades de aprendizaje de los alumnos como base para su actuación docente 
- organizar y dirigir situaciones de aprendizaje, utilizando al contexto sociopolítico, sociocultural y sociolingüístico como fuente de enseñanza

- concebir y desarrollar dispositivos pedagógicos para la diversidad asentados sobre la confianza en las posibilidades de aprender de los alumnos

- involucrar activamente a los alumnos en sus aprendizajes y en su trabajo

- acompañar el avance en el aprendizaje de los alumnos identificando tanto los factores que lo potencian como los obstáculos que constituyen dificultades para el aprender

- tomar decisiones sobre la administración de los tiempos y el ambiente del aula para permitir el logro de aprendizajes del conjunto de los alumnos

- conducir los procesos grupales y facilitar el aprendizaje individual

- reconocer y utilizar los recursos disponibles en la escuela para su aprovechamiento en la enseñanza

- seleccionar y utilizar nuevas tecnologías de manera contextualizada

- reconocer las características y necesidades del contexto inmediato y mediato de la escuela y de las familias

- participar en el intercambio y comunicación con los familias para retroalimentar su propia tarea

- trabajar en equipo con otros docentes, elaborar proyectos institucionales compartidos y participar y proponer actividades propias de la escuela" (CFCyE, Res 24/07: 8-9)

El DC para el Profesorado de Educación Primaria 2007 estableció una serie de modificaciones, entre las cuales queremos destacar:

- La duración de la carrera, que se extiende de tres a cuatro años.

- El campo de práctica docente incluye dos ejes, la "Prácticas en terreno" y las "Herramientas de la práctica", que se configuran como ejes transversales de todos los años, focalizado en diversos espacios sociales, no sólo en el escolar.

- Se genera el Taller de Integración Interdisciplinar (TAIN), como un espacio curricular transversal a todas las materias en cada uno de los años, compuesto por 4 horas reloj mensuales, con el objeto de brindar tratamiento a las diversas problemáticas y/o temáticas que cada institución y/o estudiantes y docentes consideren oportunas tratar en función del eje mencionado para cada año de la carrera.

"Se puede plantear una característica particular del diseño de la Provincia de Buenos Aires. Fue un reclamo histórico que los docentes de la formación inicial tenían, que era tener tiempo de trabajo con los estudiantes y con los docentes de otras áreas o campos del conocimiento. Por lo tanto, esos diseños integran a los Talleres Integradores Interdisciplinarios [TAIN]. Es el trabajo compartido de docentes y estudiantes a lo largo de todo el ciclo lectivo y de todos los años de formación” (Entrevista a Directora de Educación Superior). 
"El diseño curricular está organizado alrededor de cinco Campos y Trayectos opcionales, vinculados por preguntas centrales y organizadores de relaciones entre las diferentes Materias que los componen:

- Campo de Actualización Formativa. ¿Qué aspectos de la formación previa es necesario profundizar para transitar la formación docente?

- Campo de la Fundamentación. ¿Cuál es el sentido de la docencia en el mundo actual en la sociedad latinoamericana y argentina?

- Campo de la Subjetividad y las Culturas. ¿Qué saberes permiten el reconocimiento y la comprensión del mundo subjetivo y cultural del sujeto de la educación?

- Campo de los Saberes a Enseñar. ¿Cuáles son los núcleos de saberes significativos y socialmente productivos que se articulan en la enseñanza?

- Campo de la Práctica Docente. ¿Cuáles son los recorridos formativos necesarios para asumir una praxis transformadora de la práctica docente?

- Trayectos Formativos Opcionales. ¿Cuáles son los recorridos complementarios de la formación que percibe y propone cada Institución?" (DC, 2007: 27 y sig.).

De acuerdo a la incidencia de Jorge Huergo en su rol de Director de Educación Superior (DES) durante este proceso en el periodo 2005-2007, resulta productivo recuperar su concepción sobre la gestión pública. Huergo (2010) sostiene dos observaciones por un lado, se relaciona con la guerra y su finalidad, retomando los términos de Paulo Freire, es el exterminio del otro, su disolución. En cambio, en la segunda acepción, apoyando en los planteos freireanos discute con la noción empresarial de "gestionar", apoyada desde lógicas mercantilistas. De acuerdo con ello, Huergo supone que la gestión es (y debe ser) un producto colectivo: una acción y un "hacer con otros". Es decir, entiende la gestión en relación a la constitución de identidades, las relaciones de poder en ella implicadas y las diversas articulaciones necesarias para construir hegemonía. En sus palabras, la gestión es

“[...] un proceso de construcción colectiva desde las identidades, las experiencias y las habilidades de quienes allí participan. Esto quiere decir que el proceso de gestión no debe apuntar a la negación o aplanamiento de diferencias, o al acallamiento de conflictos; sino que necesariamente debe articularlos, construyendo procesos colectivos, donde lo 'colectivo' no es lo homogéneo, sino una plataforma y un horizonte común, una trama de diferencias articuladas en una concreción social. Lo que implica el reconocimiento y la producción de una cultura colectiva, organizacional o institucional [...] La gestión implica también una concepción y una práctica respecto del poder, de la administración y la circulación del mismo y de las formas de construir consensos y hegemonías dentro de una determinada organización o institución. Vale recalcar que la construcción de hegemonías (según lo expresan los filósofos Ernesto Laclau y Chantal Mouffe) no significa inmediatamente el planteamiento de situaciones de dominio, sino la posibilidad y el proyecto de articulación de fuerzas y de diferencias, a través de 
un imaginario y un objetivo común. Por eso, gestionar es más que conducir. (Huergo, 2010: 3).

Esta concepción tuvo un impacto significativo en lo que se propuso en el MGPC. Asimismo, es factible encontrar la concepción del currículo desarrollado por Alicia de Alba (1995) en relación con los temas sociales que intervienen en él. De acuerdo con el análisis de los borradores que formaron el proceso de consulta para la redefinición del currículo, consideramos que las contribuciones del autor pueden evidenciarse al menos de dos maneras: la participación de tres tipos de asignaturas, es decir, asignaturas de determinación curricular, asignaturas de estructuración formal. del plan de estudios y las asignaturas de desarrollo curricular, así como sus funciones específicas, pero, en segundo lugar, y tal vez esta dimensión es la que se vuelve más relevante, es que las funciones en sí mismas están determinadas para cada una de las asignaturas y están relacionadas entre sí. Consideremos más explícitamente esta afirmación: si bien de Alba plantea, claro está, el curriculum como un conjunto de negociaciones, puede leerse que los sujetos intervinientes poseen distintos niveles de participación [Estado, instituciones, prácticas cotidianas -docentes y estudiantes en el espacio cotidiano del aula-] en el caso particular que nos ocupa, puede sostenerse que mediante el proceso de consulta promovido desde el Estado Provincial (sujeto de determinación curricular), las funciones de los sujetos de estructuración y desarrollo curricular se producen en el mismo nivel de significación, negociación e importancia. De este modo, la consulta se configuró como un proceso de retroalimentación entre los sujetos de determinación curricular, los sujetos de estructuración formal del curriculum y los sujetos de desarrollo curricular. Parece existir allí una orientación que apela a la construcción participativa y con cierto nivel de horizontalidad en la toma de decisiones en el proceso de redefinición curricular.

De las implicancias de estos procesos es muy clara la narración que testimonia quien era en ese momento Directora Provincial de Educación Superior y Capacitación Educativa:

"En ese momento ocurre algo grave que es el hecho de que nosotros teníamos muy avanzado el proceso de construcción curricular, no exento de todas estas tensiones con algunos grupos que estaban realmente "de punta". Se dio que estuvo antes que la Resolución 24/07, es decir, nuestros diseños estaban antes que los lineamientos federales y era un problemón porque producir lineamientos federales se hacía por primera vez en la historia. Implicaba generar un sistema nacional de formación docente que fijara los lineamientos fundamentales. [...] Nuestro diseño era realmente disruptivo, desde la técnica de escritura curricular hasta el marco político pedagógico."

Planteado desde la comprensión del contexto social consecuente de la crisis social y política de 2001, los "Documentos Bases de Discusión”: DB I, DB II y DB III, enviados a los ISFD suponen un posicionamiento que rechaza el "relato único" desarrollado por el neoliberalismo, lo cual implica la necesidad de invitar y hacer partícipes a los actores involucrados en el proceso de definición curricular, bajo un proceso basado en el diálogo. 
Esto nos orienta, necesariamente, a otro aspecto señalado con el que comprendemos la redefinición de la política curricular: la concepción de la educación como acto político.

"La propuesta de redefinición curricular no va en el sentido de una fundación o una innovación que desconozca a los sujetos y los procesos históricos de nuestra educación. Ni siquiera en el sentido de una 'reforma', que tipificó la etapa anterior del estado neoliberal. La propuesta pretendía recoger y resignificar matrices de pensamiento, de política educativa y de prácticas pedagógicas que fueron experimentadas en nuestro país y en América Latina, con un sentido popular, crítico y liberador.” (DGCyE -DES, DB I, 2006: 16).

Se hace evidente allí, la noción del curriculum como proceso de desarrollo participativo pero también el destacado de un posicionamiento docente, asociado a la construcción de los saberes que realizan los docentes dentro de determinadas condiciones de posibilidad para que ello ocurra. En este sentido, se procura explicar la concepción no sólo acerca del rol docente, sino también de la educación como acto político y una concepción de la docencia que procura acercarse a la noción de "tradiciones docentes" que se consideró dentro de dicho proceso de redefinición curricular, así como el rol del Estado, las políticas públicas y la función de los asesores, expertos en educación, que fueron convocados para tal fin.

Vinculado con esta noción, en los últimos años hemos venido desarrollando el concepto de posición docente (Southwell, 2008, 2013, 2015). Cuando nos preguntarnos acerca de qué hace la diferencia de las trayectorias de formación que ofrecen unas y otras instituciones, partimos de retomar valiosas indagaciones sobre las regulaciones del trabajo docente en los estudios de historia, política y sociología de la educación en los últimos años. Desde diversos marcos teóricos y epistemológicos, un vasto número de investigaciones se han dedicado a analizar la cuestión para aproximarse a dar cuenta de problemas tales como las relaciones entre educación escolar y reproducción social y cultural, los vínculos entre el trabajo docente y la producción o el combate de la desigualdad, las disputas en torno de derechos laborales de los profesores, las diversas orientaciones del Estado, etc. Desde estos ricos y necesarios abordajes, hemos ido buscando incorporar otras dimensiones para seguir aproximándonos a esa pregunta -siempre abierta- sobre ¿qué hace la diferencia?. Cuando notamos como en instituciones similares, que atienden a matrículas muy similares, que trabajan con los obstáculos y las posibilidades comunes, con docentes igualmente regulados y con condiciones salariales iguales, habilitan trayectos formativos sustantivamente distintos para sus estudiantes.

En esa búsqueda hemos abordado el modo en que los y las educadores construyen posiciones docentes en el trabajo cotidiano con situaciones de desigualdad social y educativa, que suponen una particular lectura de esos problemas y la configuración de identidades que organizan relaciones, dinámicas y estrategias para su resolución, a partir de concepciones específicas respecto del sentido de su tarea, la presencia de elementos históricos de la profesión, y la presencia de sentidos utópicos y democratizadores que se hibridan y articulan precaria y paradójicamente con otros paternalistas o normalizadores (Southwell y 
Vassiliades, 2014). La idea de posición docente recoge un conjunto de revisiones que movimientos como el "giro cultural" o "hermenéutico" y "giro discursivo" incluyeron en el campo de la investigación social y educativa. En particular, se funda en la necesidad de considerar de forma central la construcción de significaciones por parte de los sujetos y de superar los análisis apriorísticos de la subjetividad, despegándola de ser un efecto de determinaciones de algún tipo. Por un lado, la noción de posición docente se funda en la idea de que la enseñanza supone el establecimiento de una relación con la cultura que no está situada en coordenadas predefinidas, fijas y definitivas. Esta relación supone vínculos con los saberes y las formas de su enseñanza que nunca se encuentra del todo estabilizada, al sufrir alteraciones motorizadas por la búsqueda e invención de respuestas en el contexto de los procesos de escolarización.

Hablar de sujeto remite a posiciones de sujeto en el interior de un discurso. Los sujetos no constituyen el origen de las relaciones sociales, ya que la experiencia depende de condiciones discursivas de posibilidad (Laclau y Mouffe, 1987). Señalan que es imprescindible tener en cuenta que las posiciones de sujeto se configuran una serie de posiciones diferenciales. En este marco, la categoría de posición docente se refiere, así, a una construcción que se da en la relación, no siendo posible definirla, establecerla y asirla de antemano y desgajada de la construcción histórica de dicho trabajo. Se compone de la circulación de sentidos y de los discursos que regulan y organizan el trabajo de enseñar y, específicamente, a los múltiples modos en que los enseñantes asumen, viven y piensan su tarea y los problemas, desafíos y utopías que se plantean en torno de ella. La construcción de una posición docente implica formas de sensibilidad y modos en que los y las docentes se dejan interpelar por las situaciones y los otros con los que trabajan cotidianamente, como así también por las vías a través de las cuales intentan ponerse a disposición, desarrollan su trabajo de enseñanza y prefiguran vínculos que no están previamente establecidos sino que se construyen en la relación. Asimismo, esta conceptualización acentua la dimensión de posición activa, decisional, dilemática, inherentemente política en posiciones que se desarrollan en cada situación (Southwell, 2013).

\section{Contorno social, horizontes formativos y praxis: nociones en interpelaión y reflexión}

Como hemos mencionado, el Plan de Trabajo propuesto a los ISFD, no se redujo a una serie de líneas de acciones verticales desde los niveles gubernamentales, con el asesoramiento de especialistas para concluir en una implementación de las determinadas acciones. Por el contrario, supuso un proceso circular de consulta, orientado por los funcionarios de la Dirección de Educación Superior de la provincia de Buenos Aires.

En las entrevistas realizadas a docentes y autoridades de los IFDs se evidencian las tensiones, las disputas y las interpretaciones que la redefinición curricular suponía. 
"Muchos docentes de este instituto fueron convocados básicamente en el área de las didácticas específicas, (...) muchos interpretaron que era una discusión que en realidad estaba definida antes, esto fue lo que pasó en esta institución" (Directora de la Región 1).

"Trabajé en formación docente por más de 20 años y en un instituto en particular, 22 años. Con lo cual pasé por distintos planes de estudio sobre todo en el Profesorado de Educación Primaria y cuando se hace este cambio, hay algo que resulta absolutamente novedoso que se da ahí y es que no se plantea sólo en el cambio curricular en términos de currículum, de la prescripción del plan de estudios, sino que hubo un proceso de acompañamiento a la implementación curricular. Yo fui parte de eso, se inicia con el primer año [de la carrera] y a medida que avanzan se va acompañando a lo largo de los años. Lo más novedoso en este borrador era que la práctica docente como vertebrador de la formación (...) La diferencia es que acá lo que se planteaba era una idea de práctica docente que no era solo lo estrictamente escolarizado, además de lo formal educación popular y tratando de trabajar algo de eso" (Entrevista a Docente Campo de la Práctica Región 11).

"Siempre que aparece una reforma curricular se generan expectativas e inseguridades. No todos están de acuerdo en que una reforma curricular es un desafío que hay que afrontar [...] Por ejemplo, los profesores estuvimos todos un tanto en desacuerdo en el tema de considerar al docente como un animador cultural" (Entrevista a Directora de la Región 4).

El documento que finalmente configuró el DC se caracteriza por plantearse como un documento que, sin perder su condición prescriptiva, posee una marcada y profunda fundamentación teórica, donde pueden leerse particularmente los aspectos analizados en este trabajo. En el DC cobró fuerza la categoría "horizonte formativo", la misma se presenta como una noción amplia y abierta, dentro de la cual se pueden evidenciar tres claros sentidos en relación al rol docente: el docente como trabajador de la cultura, como pedagogo y como profesional de la enseñanza. Tal como expresa el propio marco curricular:

"La noción de horizonte formativo alude a esa zona de imaginación en la tensión entre un sujeto real y tendencial (si la tendencia al futuro fuera "natural") y un sujeto deseado (siempre imposible). Como noción histórico-prospectiva, toma distancia de una mera enumeración de "competencias", así como de la idea de perfil de formación, en la medida en que pone énfasis en un proceso de construcción en un campo educativo condicionado histórica y socioculturalmente, lleno de tensiones e incertidumbres, complejo y cambiante, marcado por relaciones intersubjetivas y por encuadres institucionales, más que en la realización de una imagen ya cristalizada de antemano que se caracteriza generalmente por una serie de competencias.

Por ello al proponerse horizontes formativos, el currículum posee efectos de sentido que son, fundamentalmente, efectos de producción de identidades sociales 
particulares (...) Por eso se opta por hablar no sólo de un maestro/a profesional de la enseñanza, sino también de un maestro/a pedagogo y también trabajador cultural (transmisor, movilizador y creador cultural). En todos los casos se alude a un trabajador de la educación, a un trabajador del campo intelectual. (...)

El docente es hacedor de cultura y tiene que ser hacedor del discurso sobre las culturas y la educación.

En el horizonte formativo se reconocen tres propósitos:

- el fortalecimiento de la identidad y la significación social de la profesión docente;

- la construcción del maestro/a como productor colectivo del discurso pedagógico;

- el posicionamiento activo del maestro/a como trabajador de la cultura" (DGCyE- DES-DC: 15-16).

En segundo lugar, la participación de la comunidad educativa en la reformulación de la política curricular interpela a los sujetos que se encuentran en formación a ser sujetos con voz propia en la definición de su propia tarea y rol, en tanto se reconocen los distintos campos de significación de los sujetos involucrados en el acto educativo. En relación a los docentes en el proceso de definición curricular:

“[...] la concepción relacional del sujeto pedagógico plantea como ineludible considerar al docente como agente activo e irremplazable para la toma de decisiones curriculares. En este sentido, la mediación que los/as docentes hacen al interpretar las indicaciones curriculares, al descontextualizar y recontextualizar los saberes propios de las distintas disciplinas -producidos por otros/as en otros contextos y que son patrimonio y legado común- para re-crearlos y transmitirlos, los/as ubica como profesionales críticos/as capaces de dar sentido a su diario accionar. El/la docente es productor/a de conocimiento, un conocimiento relacionado con la transmisión cultural y la generación de condiciones que hacen posibles los aprendizajes en cada uno de los contextos específicos" (DGCyEDES-MGPC, 2007:20).

De este modo, en el Documento Base II de Discusión: "contexto y fundamentación", entiende al sujeto en formación al que se interpela como un sujeto complejo, distando de considerarlo como un sujeto que retransmite linealmente los acuerdos curriculares abordados. De este modo, supone:

"El verdadero texto curricular [...] es aquel que se construye en el encuentro entre dos horizontes de significación: el de la propuesta y los agentes que la llevan adelante, la administran, la gobiernan y la enseñan, y el de quienes están en proceso de formación. Sin embargo, ambos comparten un mismo contexto sociocultural: en él están inmersos y de él devienen las posibilidades de significación" (DES, DB II, 2006: 16). 
Cuando se apela a la noción de interpelación, aludimos, entre otros, a las significaciones otorgadas por Stuart Hall (1996). El autor es muy claro en relación a la distinción entre identidad e identificación, donde la interpelación cobra un rol central. De este modo, comprende la noción "identidad" como "punto de sutura" o "punto de encuentro" entre los discursos que intentan "interpelarnos" o ubicarnos en un lugar particular en tanto sujetos sociales de discursos particulares y por otro lado, por los procesos de construcción de subjetividades, es decir, los procesos "[...] que nos construyen como sujetos susceptibles de «decirse». De tal modo, las identidades son puntos de adhesión temporaria a las posiciones subjetivas que nos construyen las prácticas discursivas [...]" (Hall, 1996: 20). De este modo, el autor supone que las identidades son el resultado exitoso de una "articulación o encadenamiento" en el discurso.

"La idea de que una sutura eficaz del sujeto a una posición subjetiva requiere no sólo que aquel sea «convocado», sino que resulte investido en la posición, significa que la sutura debe pensarse como una articulación y no como un proceso unilateral, y esto, a su vez, pone firmemente la identificación, si no las identidades, en la agenda teórica" (Hall, 1996: 21).

En este sentido, es interesante recuperar una de las respuestas proporcionadas por los ISFD, en relación estrictamente a la configuración del rol docente como sujeto promotor de la cultura. La directora de la Región 4 indicaba que en relación al docente como "trabajador de la cultura", el sentido con el cual se pensó dicha categoría en una primera lectura, tuvo que ver con la noción de "animador cultural" y ello preocupaba a la comunidad educativa de esa institución en particular, especialmente al desarrollo de la formación de un docente. Asimismo, también explicita que luego de un tiempo y de diversas aclaraciones y acciones específicas, esas preocupaciones se fueron diluyendo. En sus palabras:

"Nosotros siempre habíamos sido como institución muy celosos de formar docentes para ese o aquel nivel. Entonces nunca digamos que la educación es una práctica social sino entendíamos que nuestra función específica ante la sociedad era formar docentes. Y que la cultura es un sector muy amplio que lo compartimos con muchos agentes educadores sociales. Entonces nosotros en ese lugar de profesional de la enseñanza y de formadores de formadores, nos pareció que se estaban confundiendo por ejemplo con el cine, con el teatro, con la literatura, con las sociedades de fomento, con el deporte. [...] esa especie de resquemor que generó el rol de animador cultural. A nosotros nos costó mucho entender eso. A mí me parece muy bien que los alumnos en primer año trabajen, un poco descubran o redescubran la comunidad como un espacio educador, que vean instituciones específicas donde se educa de otra manera que no sea la escuela (Entrevista a Directora de Región 4).

La entrevistada realiza referencia al Campo de la Práctica 1, correspondiente al primer año de la carrera cuyo eje es, según lo prescripto por el DC, "Ciudad educadora" y en donde 
los estudiantes transitan por espacios distintos a las instituciones educativas, vale decir, no se insertan en el espacio de la práctica en instituciones del sistema educativo formal.

Este ejemplo nos permite afirmar la complejidad del proceso de redefinición curricular, pero, fundamentalmente, la diferenciación en los "elementos" que la interpelación contiene y la adhesión o "punto de sutura" que los docentes realizan a los mismos. Para esclarecer esta afirmación debemos realizar dos observaciones. Por un lado, acerca del rol de "trabajador cultural" permitiría afirmar que los docentes pretenden ciertas especificaciones en cuanto al rol que debe configurar el proceso de formación, pero, por otro lado y del mismo modo, se asume como elemento positivo el carácter abierto y político de la categoría "horizonte formativo".

\begin{abstract}
"Las tensiones estaban dadas en las miradas, en el posicionamiento acerca de qué implicaba formar a un docente para la educación primaria. (...) Cuando dicen que la enseñanza es un proceso apolítico, con la enseñanza no se debe hacer política, esa es una de las tensiones, una discusión que fue fuerte en las instituciones. (...) Otra tensión que también surgió fue por los porcentajes de los campos disciplinares, que hacían que tuviesen determinadas situaciones de conflictividad entre los docentes curricularistas areales o especialistas y los docentes del campo de la formación general. Hubo que trabajar sobre esa tensión" (Entrevista a Directora de Educación Superior).
\end{abstract}

La categoría praxis recorre ampliamente el DC y se convierte en el eje estructurante del mismo, no sólo en el espacio destinado estrictamente a la "Práctica docente" sino que también se encuentra incluido como contenido específico de materias correspondientes al cuarto año, tales como "Reflexión filosófica de la educación" y "Dimensión ético-política de la praxis docente".

En esta dirección, desde los inicios de las discusiones en relación a la Redefinición de la Formación Docente se recuperaron distintos autores tales como Henry Giroux, Peter Mc Laren, Tomaz Tadeu da Silva y Paulo Freire. Por otro lado, es importante señalar que dichos documentos de discusión se apoyan en nociones posestructuralistas y análisis específicos sobre el curriculum. Asimismo, se asume, dentro de este marco referencial de la concepción de la educación como acto político y la definición y determinación curricular desde dicha perspectiva, la importancia de las disputas de poder, asumiendo la categoría "subjetividad" cobra una real importancia.

En síntesis, podemos afirmar que la política educativa plasmada en el DC para la formación de profesores plantea una noción acerca de la educación que supone proponer espacios educativos que se constituyan en espacios democráticos, que profundicen la participación, en el diálogo fundado y propositivo.

La centralidad de la enseñanza como quehacer profesional de la docencia se evidencia tanto en el proceso de redefinición curricular como en la configuración final de los Diseños Curriculares para la Educación Superior. En este sentido nos permitimos una extensa cita del Documento Base I que fue enviado durante el proceso de consulta y formulación del DC a los ISFD por la claridad conceptual con que pueden evidenciarse, por un lado, las 
concepciones de la "práctica docente" como eje de la formación, pero, asimismo, la educación como objeto de transformación social:

"Tenemos como propósito político formativo considerar a la práctica docente como un objeto de transformación. (...) no estamos hablando de una "adaptación" con sentido pragmático a las prácticas como se dan en los ambientes de trabajo, o sea, de un "acomodamiento" al habitus. Hablamos de un proceso dialéctico de reflexión sobre la práctica y sobre la reflexión en la acción (Gimeno Sacristán y Pérez Gómez, 1992: 419) como eje de formación, que implica una doble tarea: des-aprender la práctica que los cuerpos aprendieron tempranamente, como el juego que se juega en las instituciones educativas (Edelstein, 1995: 44), esto es: una práctica alienada (por más que se la presente como "la realidad"), a la vez que des-aprender la lógica de práctica y la práctica teórica (lo que nos configura históricamente como docentes, a la vez que la teorización implícita en el hacer); y re-aprender una práctica que parte de la recuperación de la voz de los sujetos (cf. Barco, 1994: 25-26; Davini, 1995: 126)" (DB I, 2006: 27).

De acuerdo con ello, el planteo acerca de la práctica docente cuyo significante es la "praxis", articula, dentro de la política curricular, otros sentidos que conforman la identificación docente, en tanto trabajador cultural, promotor de la transformación, pero esencialmente, como sujeto con capacidad crítica.

"Considerar la práctica docente como un objeto de transformación requiere un continuo y difícil proceso de autosocioanálisis, donde se pase de la fluctuación a la articulación entre la práctica docente como objeto y la subjetivación de la práctica docente." (DC,2007: 27)

"Se pudo ir trabajando esta cuestión de praxis porque el equipo de gestión generó una praxis en la propia instancia. No hablaban de capacitación sino de acompañamiento capacitante (...). Desde ahí hay algo que se vivió, realmente se puso en acto lo que se estaba proponiendo. (Docente Campo de la Práctica Región $11)^{\prime \prime}$

\section{Algunas líneas para concluir}

El análisis de la política curricular bonaerense despliega nociones acerca de la educación que procuraron recuperar el carácter político, social y cultural de la misma, entendiéndola en el horizonte de la igualdad, en lugar de la equidad, significante con el cual se había configurado el discurso educativo de la reforma noventista. De acuerdo con ello, la idea de educación se asoció con la expansión de derechos y la democratización de la sociedad desde una perspectiva que se orientó en una noción de justicia mucho más amplia que la estrictamente jurídica. De acuerdo con ello, se produce un reforzamiento significativo de la centralidad de la enseñanza, una nueva interpretación de la función docente que se 
distanciaba de la perspectiva tecnocrática y que es claramente expresada en los lineamientos curriculares que analizamos.

En ese marco, el significante inclusión se configuró como el significante vacío que estructuró el discurso educativo, pero también como un significante que disputó y reestructuró los sentidos de la configuración discursiva previa, que se había formulado, tanto a nivel nacional como provincial, desde la tecnocracia educativa y cuyo significante era uno con historia, reactivado en distintos momentos: la "profesionalización" docente (Southwell, 2007).

La nueva política curricular de la Provincia de Buenos Aires presentó una muy extensa fundamentación teórica donde es factible observar una puesta en funcionamiento de la noción de trabajo, entendida como proceso cultural, político y pedagógico. Así, el DC se configuró en una práctica discursiva que no sólo reconocía los sujetos curriculares en un horizonte de igualdad, sino que fundamentalmente, procuró reorientar la formación docente a partir de la noción de enseñanza articulada a partir de la centralidad socio cultural, la diversidad y la intención político-pedagógica que toda propuesta educativa conlleva, pero que aquí es impulsada desde la propia política educativa y la normativa curricular.

De acuerdo con lo anterior, el DC presentó dentro de los elementos constitutivos iniciales una serie de conceptualizaciones globales: - práctica transformadora de la Formación Docente como concepto eje; • profesional de la enseñanza, pedagogo hacedor del discurso de la educación y maestro/a como hacedor de cultura y del discurso cultural como horizonte formativo que confiere la direccionalidad a la totalidad de la propuesta; • posicionamiento transformador frente a los desafíos que le plantea la problemática áulica, institucional y social; • continuum formativo que se produce en los espacios singulares de actuación dentro de los cuales adquieren preeminencia diversos conflictos; - sujetos formadores y en formación que se entrelazan en el proceso curricular; • construcción participativa del currículum.

Así, el DC apela al necesario alejamiento de las concepciones tecnocráticas y a la consideración de los sujetos como "hacedores" de las prácticas. En este sentido, supone que "[...] el docente es un actor clave para reconstruir el sentido sociopolítico de la escuela pública y a la educación pública como política cultural; para no dejar las políticas culturales en manos del mercado ni de otros espacios corporativos" (DC, 2007: 13).

De igual modo, la perspectiva del proceso estudiado propició un paradigma articulador con centralidad sociocultural y con intencionalidad político cultural. En él se privilegió la lógica articulatoria, producida por la perspectiva y el significado sociocultural. Sin desconocer la construcción teórico epistemológica de cada lógica, se pone el acento en la centralidad sociocultural del campo que las contiene y articula, y desde donde se propone avanzar en su reconocimiento. Retomando como referencia y tradición el camino recorrido por la educación en Latinoamérica, que da sentido a ese paradigma articulador con centralidad sociocultural. 


\section{Referências}

Boulan N. (2019) Políticas de formación de profesores de nivel primario en la provincia de Buenos Aires. Discursos, curriculum y procesos identificatorios (2006-2016). Tesis de Doctorado, FaHCE, UNLP.

De Alba, A. (1995) Curriculum: crisis, mito y perspectivas. Buenos Aires: Miño y Dávila.

Giroux, Henry (1988). Los profesores como intelectuales transformativos. Barcelona: Centro de Publicaciones del M.E.C. y Ediciones Paidós Ibérica.

Hall, S. (1996) “¿Quién necesita identidad?”, en: Hall, S. \& Du Gay, P. (eds.), Cuestiones de Identidad. Buenos Aires: Amorrortu.

Huergo, J. (2010) "Los procesos de gestión" y "De la escolarización a la comunicación en educación". Seminario: Comunicación y conducción de la gestión". UNIPE. Recuperado en: http://servicios.abc.gov.ar/lainstitucion/univpedagogica/especializaciones/seminario/

Laclau, E. \& Mouffe, Ch. (1985) Hegemonía y estrategia socialista. Buenos Aires: Fondo de Cultura Económica.

Laclau, E. (1996) “¿Por qué los significantes vacíos son importantes para la política?”, en Emancipación y diferencia. Buenos Aires: Ariel.

Southwell, M. (2007) "Profesionalización docente al término del siglo XX: políticas y nominaciones producidas por organismos internacionales", en Anuario de Historia de la Educación. Sociedad Argentina de Historia de la Educación. Buenos Aires: Prometeo.

Southwell M. (2008) "Política y educación: ensayos sobre la fijación del significado" en Cruz Pineda O. y Echevarría Canto L. (Coord.) Investigación educativa. Herramientas teóricas y análisis político del discurso. México: Casa editorial Juan Pablos.

Southwell, M. (2013) "El análisis político del discurso y la cuestión de la politicidad de la educación" Conferencia dictada en el X Coloquio de Análisis Político del Discurso y Educación, México: DIECINVESTAV.

Southwell M. (2015) "La construcción de posiciones docentes frente a situaciones de desigualdad social y educativa" ponencia presentada en el seminario Figuras actuales de la Segregación FLACSO, Área de Educación, diciembre de 2015, Buenos Aires.

Southwell, M. y Vassiliades, A. (2014) "El concepto de posición docente: notas conceptuales y metodológicas". Revista Educación, Lenguaje y Sociedad. Gral. Pico: Miño y Davila. Vol.VIII n ${ }^{\circ}$.

Southwell, M. y Vassiliades, A. (2016) “Articulaciones y disputas en la trama entre trabajo docente e igualdad educativa: una aproximación histórica", en Brenner, G. \& Galli, G. (Comp.) Inclusión y calidad como políticas educativas de Estado o el mérito como opción única de mercado. Buenos Aires: Stella.

Vassiliades, A. (2012) "Regulaciones del trabajo de enseñar en la provincia de Buenos Aires: posiciones docentes frente a la desigualdad social y educativa". Tesis de Doctorado. Facultad de Filosofía y Letras. UBA. 


\section{Correspondência}

Myriam Southwell: Pedagoga. Doctora por el Departamento de Gobierno de la Universidad de Essex, Inglaterra. Magister en Ciencias Sociales con orientación en educación Facultad Latinoamericana de Ciencias Sociales.

Correo electrónico: islaesmeralda@gmail.com

Noralí Boulan: Doctora en Ciencias de la Educación por la Universidad Nacional de La Plata. Profesora en Ciencias de la Educación de la Universidad Nacional de La Plata.

Correo electrónico: noraliboulan@ gmail.com

Texto publicado em Currículo sem Fronteiras com autorização das autoras. 